\title{
On the asymptotic periods of integral functions
}

By J. M. Whittaker, Pembroke College, Cambridge.

(Received 20th December, 1932. Read 20th January, 1933.)

§1. A period of a function $f(z)$ is defined to be a number $\omega(\neq 0)$ such that

$$
\Delta_{\omega}(z)=f(z+\omega)-f(z)
$$

is identically zero; and it is not a difficult matter to show that an integral function may either have no periods or else a single sequence $k \lambda, k= \pm 1, \pm 2, \ldots$

The question may be asked "Are these the only values of $\omega$ for which $\Delta_{\omega}(z)$ can be 'smaller' than $f(z)$, or can there be other values of $\omega$ for which $\Delta_{\omega}(z)$ is 'smaller' than $f(z)$, but is not identically zero?" The term "smaller" needs precise definition. If $f(z)$ is an integral or meromorphic function it is most natural to interpret it as meaning "of lower order," and it is in this sense that the word will be understood. With this interpretation it is easy to see that in fact there may be more than a single sequence $k \lambda$ of values of $\omega$ for which $\Delta_{\omega}(z)$ is smaller than $f(z)$. For example the integral function $R(z)$ defined by

$$
R(z)=\sum_{n=1}^{\infty} \frac{e^{2 \pi i n ! z}}{n ! !}
$$

is of infinite order, but if $p$ is any rational number

is of order 1 .

$$
R(z+p)-R(z)
$$

Numbers with the property under discussion will be called asymptotic periods. Their definition is then as follows:

Def. A number $\beta(\neq 0)$ is an asymptotic period of an integral or meromorphic function $f(z)$ if $\Delta_{\beta}(z)$ is of lower order than $f(z)$. The set of all asymptotic periods of $f(z)$ is denoted by $B$.

This definition includes the case of a function $f(z)$ of infinite order. $\Delta_{\beta}(z)$ must then be of finite order. It is evident that, if $\beta, \gamma$ are asymptotic periods, then $-\beta, \beta+\gamma$ have the same property. The following result is proved. 
If $f(z)$ is an integral function, $B$ is a linear set of measure zero. ${ }^{1}$

An important part in the proof is played by an extension of Guichard's theorem, that every integral function has a finite sum. As this result is of independent interest it is discussed at the beginning, instead of in its proper place in the proof of the main theorem.

Another part of the proof consists in showing that a function of order $<1$ cannot have any asymptotic periods. More generally, this is true if

$$
\frac{\log M(r)}{r} \rightarrow 0
$$

§2. The finite sum of an integral function. It was proved by Guichard $^{2}$ that if $f(z)$ is any given integral function and $\omega \neq 0$, an integral function $g(z)$ exists with the property

$$
g(z+\omega)-g(z)=f(z) .
$$

Guichard's method is not well adapted to elucidate the relation between the orders of $f(z), g(z)$, an essential matter for the application which we have in view; but by an extension of the alternative method by which Appell and Hurwitz established (2.1) it is possible to prove the following result.

Theorem I. If $f(z)$ is an integral function of finite order $\rho$, there is an integral function $g(z)$, of order $\leqq \max (1, \rho)$, for which $(2.1)$ is satisfied.

There is no loss of generality in taking $\omega=1$. Let

The function

$$
f(z)=\sum_{n=0}^{\infty} a_{n} z^{n}
$$

$$
\psi_{n, 8}(z)=\frac{n !}{2 \pi i} \int_{C_{8}} \frac{e^{2 t}}{\left(e^{t}-1\right) t^{n}} d t,
$$

when $C_{s}$ is the circle $|t|=(2 s+1) \pi$ and $s$ is an integer, has the property $^{3}$

1 Added in proof. $B$ may be a non-denumerable set. See Journal London Math. Soc., 8 (1933), 119.125.

${ }^{2}$ Guichard, 1. See Nörlund, 2, for an account of Guichard's work and also of the work of Appell and Hurwitz mentioned below.

${ }^{3}$ Nörlund, 2, 8. $\Delta f(z)=f(z+1)-f(z)$. 


$$
\Delta \psi_{n, \varepsilon}(z)=n z^{n-1}, \quad(n=1,2, \ldots),
$$

so that a solution of (2.1) (with $\omega=1$ ) is

$$
g(z)=\sum_{n=0}^{\infty} \frac{a_{n}}{n+1} \psi_{n+1, s_{n}}(z),
$$

provided that $s_{1}, s_{2}, \ldots$ can be chosen in such a way that the seriss converges uniformly in any finite region.

If $\rho<1$ it is sufficient to take $s_{1}=s_{2}=\ldots=0$, and the order of $g(z)$ is then $\leqq 1$. For, let $\rho^{-1}>\sigma>1$, so that 1

$$
\left|a_{n}\right|<n^{-n \sigma}, \quad\left(n \geqq n_{0}\right) .
$$

Now, writing $t=(2 s+1) \pi e^{i \theta}$,

$$
\begin{aligned}
& \left|\psi_{n, 8}(z)\right| \leqq \frac{n !}{2 \pi} \int_{0}^{2 \pi} \frac{e^{(2 s+1) \pi|z|}(2 s+1) \pi d \theta}{\left|e^{t}-1\right|\{(2 s+1) \pi\}^{n}} \\
& <A n ! e^{(2 s+1) \pi(|z|+1)\{2 s+1) \pi\}^{1-n},}
\end{aligned}
$$

where $A$ is independent of $n, s, z$. Hence

$$
\sum_{n=0}^{\infty}\left|\frac{a_{n} \psi_{n+1,0}(z)}{n+1}\right|<K \sum_{n=1}^{\infty} n^{n(1-\sigma)} e^{\pi|z|}<K e^{\pi|z|} .
$$

$K$ always denotes a constant, but not always the same constant. Denoting by $M_{0}(r)$ the maximum of $|g(z)|$ for $|z|=r$, the last inequality shows that

$$
\varlimsup_{r \rightarrow \infty} \frac{\log M_{0}(r)}{r} \leqq \pi,
$$

so that $g(z)$ is of order $\leqq 1$.

If $\rho \geqq 1$, consider the function $\sigma(n)$ defined by

$$
\sigma(n)=\min _{m \xi^{\prime} n}\left(\frac{\log \left|a_{m}\right|^{-1}}{m \log m}\right) .
$$

$\sigma(n)$ is an increasing function, and

$$
\sigma(n) \rightarrow \lim _{n \rightarrow \infty} \frac{\log \left|a_{n}\right|^{-1}}{n \log n}=\frac{1}{\rho} .
$$

Again, writing $a(n)=1-\sigma(n)$,

$$
n^{n a(n)}\left\{\left(2 s_{n}+1\right) \pi\right\}^{-n}<e^{-n}, \quad(n \geqq 1),
$$

provided that

and this is so if

$$
\left(2 s_{n}+1\right) \pi>e n^{a(n)},
$$

$$
s_{n}=\left[n^{a(n)}\right] \text {. }
$$

\footnotetext{
2 Valiron, 4, 40
} 
It will be shown that with this choice of $s_{1}, s_{2}, \ldots,(2.5)$ defines an integral function of order $\rho$.

Corresponding to a given value of $r=|z|$ define the integer

$$
N(r)=\left[(8 r)^{1 / \sigma(2)}\right] .
$$

Then (2.7), (2.9), (2.11), (2.12) show that

$$
\begin{aligned}
v_{n}(z) & =\left|\frac{a_{n} \psi_{n+1, s_{n}}(z)}{n+1}\right| \\
& <n^{-n \sigma(n)} n^{n}\left\{\left(2 s_{n}+1\right) \pi\right\}^{-n} \exp \left\{\left(2 s_{n}+1\right) \pi r\right\} \\
& <\exp \left\{-n+7 n^{\alpha(n)} r\right\} \\
& <e^{-n / 8}, \quad(n>N),
\end{aligned}
$$

since

$$
\begin{aligned}
n-7 n^{a(n)} r-\frac{n}{8} & >\frac{7}{8} n-\frac{7}{8} n^{a(n)+\sigma(2)} \\
& =\frac{7}{8} n\left\{1-n^{\sigma(2)-\sigma(n)}\right\} \geqq 0 .
\end{aligned}
$$

Now take a fixed integer $p$ and write $a(p)=\alpha$. Then

$$
\begin{aligned}
\sum_{n=p}^{N} v_{n}(z) & <\sum_{n=p}^{N} \exp \left\{-n+7 n^{a(n)} r\right\} \\
& \leqq \sum_{n=p}^{N} \exp \left\{-n+7 n^{a} r\right\} \\
& <N(r) \mu(r),
\end{aligned}
$$

where $\mu(r)$ is the greatest term of the last series. $\mu(r)$ is determined by finding the maximum value of the function

namely

$$
-x+7 x^{a} r
$$

so that

$$
\left(\frac{1}{a}-1\right)(7 a r)^{1 /(1-a)}
$$

$$
\mu(r) \leqq \exp \left\{\left(\frac{1}{a}-1\right)(7 a r)^{1 /(1-a)}\right\} .
$$

Now, making use of (2.14); (2.15),

$$
M_{0}(r)<\sum_{n=0}^{p} v_{n}(z)+N(r) \mu(r)+\sum_{n=N+1}^{\infty} e^{-n / 8},
$$


whence, by (2.13), (2.16),

$$
\varlimsup_{r \rightarrow \infty} \frac{\log \log M_{0}(r)}{\log r} \leqq(1-a)^{-1}=\{\sigma(p)\}^{-1}
$$

Finally, make $p$ tend to infinity, and use (2.10).

It follows that $g(z)$ is of order $\leqq \rho$. On the other hand it is evident that $g(z)$ is of order $\geqq \rho$, so that $g(z)$ must be of order $\rho$.

§3. THEOREM 2. An integral function has no asymptotic periods if

or if

$$
\begin{aligned}
& \varlimsup_{r \rightarrow \infty} \frac{\log M(r)}{r}=0, \\
& \varlimsup_{r \rightarrow \infty} \frac{(\log r)^{2} \log M(r)}{r}=0 .
\end{aligned}
$$

The importance of a hypothesis such as (3.2), involving a lower limit instead of an upper limit, will be seen at a later stage of the proof of the main theorem.

We need three lemmas of Phragmén-Lindelöf type.

LEMMA 1. An integral function is a constant if it satisfies the conditions

$$
\begin{aligned}
\varlimsup_{r \rightarrow \infty} & \frac{\log M(r)}{\sqrt{ } r}=0, \\
& |f(-x)| \leqq C, \quad(x \geqq 0) .
\end{aligned}
$$

This result is due to Pólya and Szegö. ${ }^{1}$

Lemma 2. In Lemma 1 (3.3) may be replaced by

The function

$$
\lim _{r \rightarrow \infty} \frac{(\log r)^{2} \log M(r)}{\sqrt{ } r}=0 .
$$

$$
h(z)=f(z) \exp \left(-\frac{\sigma z^{\frac{1}{2}}}{\log z}\right), \quad \cdot(\sigma>0),
$$

is regular in the domain $D$ defined by

$$
100<r<R, \quad-\pi<\theta<\pi, \quad\left(z=r e^{i \theta}\right) .
$$

1 Polya and Szegö, 3, 149. 
$z^{\frac{1}{3}} / \log z$ is taken to be the branch which is real and positive on the real positive axis. Now

and

$$
\left|\exp \left(-\frac{\sigma z^{\frac{1}{3}}}{\log z}\right)\right|=\exp \left\{-\frac{\sigma \sqrt{ } r\left(\log r \cos \frac{1}{2} \theta+\theta \sin \frac{1}{2} \theta\right)}{(\log r)^{2}+\theta^{2}}\right\},
$$

so that in $D$

$$
\left.\begin{array}{rlrl}
\cos \frac{1}{2} \theta \log r+\theta \sin \frac{1}{2} \theta & \geqq \frac{1}{\sqrt{ } 2} \log r, & & \left(0 \leqq \theta \leqq \frac{\pi}{2}\right), \\
& \geqq \frac{\pi}{2 \sqrt{ } 2}, & \left(\frac{\pi}{2} \leqq \theta \leqq \pi\right),
\end{array}\right\}
$$

$$
|h(z)| \leqq|f(z)| \exp \left\{-\frac{\sigma \sqrt{ } r}{2(\log r)^{2}}\right\} .
$$

By (3.5), $R$ may be chosen so that

$$
\log M(R)<\frac{\sigma \sqrt{ } R}{2(\log R)^{2}} .
$$

Hence, by the maximum modulus principle,

$$
|h(z)| \leqq \max \{C, 1, M(100)\}
$$

for all points $z$ in $D$. Finally, keeping $z$ fixed, let $\sigma \rightarrow 0$. It follows that, for all values of $z$,

$$
|f(z)| \leqq \max \{C, 1, M(100)\},
$$

and hence $f(z)$ is a constant.

LEMMA 3. If an integral function $f(z)$ satisfies either (3.3) or (3.5) and

$$
|f(-x)| \leqq C e^{x^{\alpha}}, \quad\left(0<a<\frac{1}{2}\right), \quad(x \geqq 0),
$$

there is a constant $A$ such that for all values of $z$

$$
|f(z)| \leqq A e^{r^{\alpha}}
$$

It is only necessary to apply the arguments of the preceding lemmas to the function

$$
f(z) \exp \left\{-(-z)^{a}\right\} \text {. }
$$

Theorem 2 is a simple deduction. Suppose, if possible, that a function $f(z)$ of order $\rho$ satisfies either (3.1) or (3.2) and has an asymptotic period $\beta$, so that $\Delta_{\beta}(z)$ is of order $\sigma<\rho$. In the former case $\rho \leqq 1$, but in the latter $\rho$ may be any finite number or infinity. There is no loss of generality in taking $\beta$ real and positive. Moreover we may suppose that the zeros $a_{1}, a_{2}, \ldots$, of $f(z)$ have $\rho$ as 
exponent of convergence. This can always be secured by adding a suitable constant to $f(z)$. Then, if $0 \leqq t \leqq \beta$,

so that

$$
\begin{aligned}
& f(t+n \beta)=f(t)+\sum_{\delta=0}^{n-1} \Delta_{\beta}(t+s \beta), \\
& f(t-n \beta)=f(t)-\sum_{\delta=1}^{n} \Delta_{\beta}(t-s \beta),
\end{aligned}
$$

$$
|f(t \pm n \beta)| \leqq M(\beta)+n M_{\beta}\{(1+n) \beta\},
$$

when $M_{\beta}(r)$ denotes the maximum modulus of $\Delta_{\beta}(z)$. Hence if $\sigma<2 \tau<\rho$ and $x \geqq 0$,

$$
\begin{aligned}
|f( \pm x)| & \leqq M(\beta)+\frac{2 x}{\beta} M_{\beta}(2 x) \\
& <K e^{x^{2 \tau}}
\end{aligned}
$$

and so the function $h(z)$ defined by

is such that

$$
h\left(z^{2}\right)=f(z) f(-z)
$$

$$
|h(x)|=|f(\sqrt{ } x) f(-\sqrt{ } x)|<K^{2} e^{2 x^{r}}, \quad(x \geqq 0) .
$$

Now $h(z)$ satisfies (3.3) or (3.5) according as $f(z)$ satisfies (3.1) or (3.2), and it follows from Lemma 3 that the order of $h(z)$ cannot exceed $\tau$.

On the other hand the zeros of $h(z)$ are $a_{1}^{2}, a_{2}^{2}, \ldots$ and these have exponent of convergence $\rho / 2$. Hence the order of $h(z)$ cannot be less than $\rho / 2$. The contradiction implies that $f(z)$ has no asymptotic periods.

\$4. Lemma 4. The ratio of two asymptotic periods of an integral function is real.

If possible, let $\beta, \gamma$ be asymptotic periods whose ratio is not real. It follows from the identity

$$
f(z+k \beta+l \gamma)=f(z)+\sum_{s=0}^{l-1} \Delta_{\gamma}(z+s \gamma)+\sum_{t=0}^{k-1} \Delta_{\beta}(z+l \gamma+t \beta)
$$

that if $|z|=r$,

$$
f(z)=f\left(z_{0}\right)+\sum_{s} \Delta_{\gamma}\left(z_{8}\right)+\sum_{t} \Delta_{\beta}\left(z_{t}^{\prime}\right),
$$

where $z_{0}$ is in a "period parallelogram" with the origin as one vertex, $z_{1}, z_{2}, \ldots$ are points inside the circle $|z|=r$, each in a different 
" period parallelogram," and the same is true of $z_{1}^{\prime}, z_{2}^{\prime}, \ldots$ In each case the number of points does not exceed $K(\beta, \gamma) r$. Hence

$$
M(r) \leqq M(|\beta|+|\gamma|)+K(\beta, \gamma) r\left\{M_{\beta}(r)+M_{\gamma}(r)\right\},
$$

and this is evidently incompatible with the hypothesis that $\Delta_{\beta}(z), \Delta_{\gamma}(z)$ are of lower order than $f(z)$.

§5. LEMMA 5. Let $f_{1}(z), f_{2}(z)$ be periodic integral functions of order $\rho$, whose periods $\omega, \omega / \mu$ are real, and let

$$
g(z)=f_{1}(z)-f_{2}(z), \quad z=x+i y .
$$

If, for some $\tau<\rho$,

$$
|g(x+i y)|<K e^{|y|^{\top}}, \quad(|y| \geqq a),
$$

where $K$ is independent of $x$, then $\mu$ is a rational number.

$\rho$ may be $\infty$. There is no loss of generality in taking $\omega=2 \pi$. If $\mu$ is not rational, consider the function $f_{1}(-i \log w)$, any branch of the logarithm being chosen. This function is regular and uniform in every annulus $0<R \leqq|w| \leqq R^{\prime}$, and so can be expanded in a Laurent series

so that, writing $z=-i \log w$,

$$
\sum_{n=0}^{\infty} a_{n} w^{n}+\sum_{n=1}^{\infty} \dot{b}_{n} w^{-n}
$$

In the same way

$$
f_{1}(z)=\sum_{n=0}^{\infty} a_{n} e^{n i z}+\sum_{n=1}^{\infty} b_{n} e^{-n i z}
$$

$$
f_{2}(z)=\sum_{n=0}^{\infty} c_{n} e^{n \mu j z}+\sum_{n=1}^{\infty} d_{n} e^{-n \mu i z} \text {. }
$$

As $f_{1}(z)$ is of order $\rho$ at least one of the series in (5.2), say the first, is an integral function of order $\rho$.

Now, as $\mu$ is irrational, the exponentials in (5.2), (5.3) are all distinct, and so

and thus, if $y \geqq 0$,

$$
a_{n} e^{n i z}=\lim _{T \rightarrow \infty} \frac{1}{2 T} \int_{-T}^{T} e^{n i x-t)} g(t+i y) d t
$$

$$
\begin{aligned}
\left|a_{n}\right| e^{n y} & \leqq \varlimsup_{T \rightarrow \infty} \frac{1}{2 T} \int_{-T}^{T}|g(t+i y)| d t \\
& \leqq G(y),
\end{aligned}
$$

where

By (5.1),

$$
G(y)=\max _{-\infty<x<\infty}|g(x \pm i y)|
$$


Now the function

$$
F(w)=\sum_{n=0}^{\infty} a_{n} w^{n}, \quad w=r_{1} e^{i \phi}
$$

is an integral function. Let $A\left(r_{1}\right)$ denote its maximum modulus,

$\gamma$ its order, and

$$
A\left(r_{1}\right)=\max _{|w|=r_{1}} \mid F(w) !,
$$

$$
a\left(r_{1}\right)=\max _{n \lesssim 0}\left|a_{n}\right| r_{1}^{n}
$$

its maximum term. (5.4) shows that

$$
a\left(e^{y}\right) \leqq G(y), \quad(y \geqq 0) .
$$

Moreover if $M_{0}(r)$ denotes the maximum modulus of $F\left(e^{i z}\right)$,

$$
M_{0}(y) \leqq A\left(e^{y}\right), \quad(y \geqq 0) .
$$

For $M_{0}(y)$ is the maximum of $|F(w)|$ on the curve

$$
\left(\log r_{1}\right)^{2}+\phi^{2}=y^{2}, \quad(-\pi \leqq \phi \leqq \pi),
$$

in the $w$-plane, and this lies inside the curve

Now if $\gamma$ is finite, 1

$$
\log r_{1}=y \text {. }
$$

$$
\frac{\log a(r)}{\log A(r)} \rightarrow 1, \quad \text { as } r \rightarrow \infty,
$$

and so by (5.6), (5.7),

Moreover

$$
\lim _{y \rightarrow \infty} \frac{\log G(y)}{\log M_{0}(y)} \geqq 1 .
$$

Hence

$$
\varlimsup_{y \rightarrow \infty} \frac{\log \log M_{0}(y)}{\log y}=\rho .
$$

$$
\varlimsup_{y \rightarrow \infty} \frac{\log \log G(y)}{\log y} \geqq \rho>\tau,
$$

and this contradicts (5.5).

Again, if $\gamma$ is infinite, ${ }^{2}$

whence by (5.6)

$$
\varlimsup_{r \rightarrow \infty} \frac{\log \log \alpha(r)}{\log r}=\infty,
$$

$$
\varlimsup_{y \rightarrow \infty} \frac{\log \log G(y)}{y}=\infty,
$$

which likewise contradicts (5.5).

1 Valiron, 4, 32.

$\because$ Cf. Valiron 4. (5.9) is not explicitly stated but follows readily from equation (2.9) on p. 31. 
§6. Functions of order $>1$. It follows from Theorem 2 that an integral function of order $<1$ cannot have any asymptotic periods. It is convenient to take next the case of a function of order $\rho>1$ (including the case $\rho=\infty$ ).

By Lemma 4 the asymptotic periods all lie on a line through the origin, say the real axis. Let $B_{\sigma}(\sigma<\rho)$, denote the set of real numbers $\beta$ such that $\Delta_{\beta}(z)$ is of order $\leqq \sigma$. The sets $B_{\sigma}$ form an increasing sequence with limit $B$.

Take a fixed value of $\sigma>1$. If $B_{\sigma}$ is not null, let $\beta$ be a member of it. In accordance with Theorem $1 \Delta_{\beta}(z)$ can be expressed as the $\beta$-difference of a function $g_{1}(z)$ of order $\leqq \sigma$, so that

$$
f(z+\beta)-f(z)=g_{1}(z+\beta)-g_{1}(z) .
$$

Thus the function

$$
f_{1}(z)=f(z)-g_{1}(z)
$$

is periodic, of period $\beta$, and of order $\rho$. Write

$$
F_{1}(y)=\max _{0 \leqq x, x^{\prime} \leqq \beta}\left|f_{1}(x+i y)-f_{1}\left(x^{\prime}+i y\right)\right|,
$$

and choose $\tau$ so that $\sigma<\tau<\rho$. There are two cases to consider. Either

$$
F_{1}(y) \leqq e^{|y|^{\tau}}, \quad(|y| \geqq a),
$$

$$
F_{1}(y)>e^{|y|^{\tau}} \text {, for arbitrarily large values of }|y| .
$$

Take the first case. Associated with $\gamma$, any other member of $B_{\sigma}$, there are functions $g_{2}(z), f_{2}(z), F_{2}(y)$ corresponding to $g_{1}(z), f_{1}(z), F_{1}(y)$. $f_{2}(z)$ has period $\gamma$, order $\rho$, and

$$
F_{2}(y)=\max _{0 \leqq x, x^{\prime} \leqq \gamma}\left|f_{2}(x+i y)-f_{2}\left(x^{\prime}+i y\right)\right| .
$$

It will be shown that (6.2) implies that

$$
F_{2}(y) \leqq 2 e^{|y|^{\tau}}, \quad(|y| \geqq b) .
$$

For consider the function $g(z)$, of order $\leqq \sigma$, defined by

$$
g(z)=f_{1}(z)-f_{2}(z)=g_{2}(z)-g_{1}(z) .
$$

If $(6.4)$ is false, then corresponding to a given value of $K$, numbers $x, x^{\prime}, y$ can be found such that

and

$$
0 \leqq x, x^{\prime} \leqq \max (\beta, \gamma), \quad|y|>K,
$$

$$
\begin{aligned}
\left|g(x+i y)-g\left(x^{\prime}+i y\right)\right| \geqq\left|f_{2}(x+i y)-f_{2}\left(x^{\prime}+i y\right)\right| & -\left|f_{1}(x+i y)-f_{1}\left(x^{\prime}+i y\right)\right|
\end{aligned}
$$


Hence, writing

$$
\begin{gathered}
M_{0}(r)=\max _{\mid z:=r}|g(z)|, \\
2 M_{0}(2|y|) \geqq|g(x+i y)|+\left|g\left(x^{\prime}+i y\right)\right|>e^{|y|^{\tau}},
\end{gathered}
$$

and this is impossible if $K$ is sufficiently large, as the order of $g(z)$ does not exceed $\sigma$.

(6.4) being thus established, it follows that if $x, x^{\prime}$ are any real numbers then

$$
\begin{aligned}
\left|g(x+i y)-g\left(x^{\prime}+i y\right)\right| & \leqq\left|f_{2}(x+i y)-f_{2}\left(x^{\prime}+i y\right)\right|+\left|f_{1}(x+i y)-f_{1}\left(x^{\prime}+i y\right)\right| \\
& \leqq F_{2}(y)+F_{1}(y) \\
& \leqq 3 e^{|y|^{\tau}, \quad(|y| \geqq c),}
\end{aligned}
$$

and so if $-\infty<x<\infty$,

$$
\begin{aligned}
|g(x+i y)| & \leqq g(i y) \mid+3 e^{|y|^{\tau}} \\
& <4 e^{|y|^{\tau}}, \quad(|y| \geqq d),
\end{aligned}
$$

since $g(z)$ is of order $\leqq \sigma<\tau$.

It now follows from Lemma 5 that $\gamma$ is a rational multiple of $\beta$.

The alternative case, expressed by (6.3), is dealt with by a different argument.

Let $N$ be a "large" positive integer and let $\sigma<\lambda<\tau$. At least one of $\pm \infty$, say $\infty$, is a limit point of the numbers $y$ for which (6.3) is satisfied. Hence there is a sequence $y_{1}, y_{2}, \ldots$, tending to $\infty$, such that

and also

$$
F_{1}\left(y_{p}\right)>\exp \left(y_{p}^{\tau}\right) \quad(p=1,2, \ldots),
$$

$$
y_{p}^{\tau}>(N+p+1) y_{p}^{\lambda} .
$$

Corresponding to $y_{p}$, a member of this sequence, let $x_{1}, x_{2}$ be points of $(0, \beta)$ for which

$$
\left|f_{1}\left(x_{1}+i y_{p}\right)-f_{1}\left(x_{2}+i y_{p}\right)\right|=F_{1}\left(y_{p}\right) .
$$

Mark in the $f_{1}$-plane the values of $f_{1}(z)$ corresponding to values of $z=x+i y_{p}, 0 \leqq x \leqq \beta$. The resultiug curve is continuous and closed, since $f_{1}(z)$ has period $\beta$, and is contained in the circle of centre $f_{1}\left(x_{1}+i y_{p}\right)$, radius $F_{1}\left(y_{p}\right)$. Now divide this circle into annuli by drawing concentric circles of radii $s F_{1}\left(y_{p}\right) / S,(s=1,2, \ldots, S-1)$, 
where $S=2^{N+p}$, and denote by $E_{8}$ the set of points $x$ for which the $f_{1}$-curve lies in the $s^{\text {th }}$ annulus. To make matters precise, let $E_{8}$ consist of the points $x$ in $(0, \beta)$ for which

$(s-1) S^{-1} F_{1}\left(y_{p}\right) \leqq\left|f_{1}\left(x_{1}+i y_{p}\right)-f_{1}\left(x+i y_{p}\right)\right|<s S^{-1} F_{1}\left(y_{p}\right),(s=1,2, \ldots, S-1)$

Evidently $\leqq F_{1}\left(y_{p}\right),(s=S)$.

$$
m E_{1}+m E_{2}+\ldots+m E_{S}=\beta,
$$

so that there is at least one value of $k$ for which

$$
m E_{k} \leqq \beta S^{-1} \text {. }
$$

As the $f_{1}$-curve is continuous, and passes from the centre of the circle of radius $F_{1}\left(y_{p}\right)$ to its circumference, it must cut the concentric circle of radius $\left(k-\frac{1}{2}\right) S^{-1} F_{1}\left(y_{p}\right)$. That is to say, there is a point $\xi_{p}$ in $(0, \beta)$ such that

$$
\left|f_{1}\left(\xi_{p}+i y_{p}\right)-f_{1}\left(x_{1}+i y_{p}\right)\right|=\left(k-\frac{1}{2}\right) S^{-1} F_{1}\left(y_{p}\right) .
$$

On drawing a diagram it is evident that if $x$ is a point of $E_{g}$, $(s \neq k)$, then

$$
\left|f_{1}\left(\xi_{p}+i y_{p}\right)-f_{1}\left(x+i y_{p}\right)\right| \geqq \frac{1}{2} S^{-1} F_{1}\left(y_{p}\right),
$$

and hence the values of $x$ in $(0, \beta)$ for which this inequality is satisfied form a set $E^{(p)}$ of measure $\geqq\left(1-S^{-1}\right) \beta$.

As $f_{1}(z)$ has period $\beta$ this means that the values of $t$ in $(0, \beta)$ for which

$$
\left|f_{1}\left(\xi_{p}+i y_{p}\right)-f_{1}\left(\xi_{p}+t+i y_{p}\right)\right| \geqq \frac{1}{2} S^{-1} F_{1}\left(y_{p}\right)
$$

form a set $T_{p}$ of measure $\geqq\left(1-S^{-1}\right) \beta$. Let

so that

$$
T=T_{1} T_{2} T_{3} \ldots
$$

$$
m T \geqq\left(1-\sum_{p=1}^{\infty} 2^{-p-N}\right) \beta=\left(1-2^{-N}\right) \beta .
$$

It will now be shown that the sets $T, B_{\sigma}$ have no members in common.

If $T B_{\sigma}$ is not null, let $\gamma$ be a member of it. $g_{2}(z), f_{2}(z), g(z)$ are defined as before, and

$$
g\left(\xi_{p}+i y_{p}+\gamma\right)-g\left(\xi_{p}+i y_{p}\right)=f_{\mathbf{1}}\left(\xi_{p}+i y_{p}+\gamma\right)-f_{1}\left(\xi_{p}+i y_{p}\right)
$$

since $f_{2}(z)$ has period $\gamma$. Hence, for all values of $p$,

$$
\begin{aligned}
2 M_{0}\left(2 y_{p}\right) & \geqq\left|g\left(\xi_{p}+i y_{p}+\gamma\right)-g\left(\xi_{p}+i y_{p}\right)\right| \\
& =\left|f_{1}\left(\xi_{p}+i y_{p}+\gamma\right)-f_{1}\left(\xi_{p}+i y_{p}\right)\right| \\
& \geqq \frac{1}{2} S^{-1} F_{1}\left(y_{p}\right)>\frac{1}{2} S^{-1} \exp y_{p}^{\tau}>\exp y_{p}^{\lambda},
\end{aligned}
$$


by (6.7), and this is false, since it implies that

$$
\varlimsup_{r \rightarrow \infty} \frac{\log \log M_{0}(r)}{\log r} \geqq \lambda>\sigma
$$

whereas $g(z)$ is of order $\leqq \sigma$. Hence the set $T B_{\sigma}$ is null, and so if $B_{\sigma}{ }^{*}$ denotes the part of $B_{\sigma}$ in the interval $(0, \beta)$, by $(6.8)$,

$$
m B_{\sigma}^{*} \leqq 2^{-v} \beta \text {. }
$$

As $N$ is independent of $\beta, \sigma$ this implies that

and so

$$
m B_{\sigma}^{*}=0
$$

$$
m B_{\sigma}=0
$$

for each $\sigma$. Hence, finally,

$$
m B=0 \text {. }
$$

§7. Functions of order 1. The remaining case, $\rho=1$, can be reduced to the one just discussed. It is enough to consider a function of regular growth i.e. such that

$$
\frac{\log }{\log M(r)} \rightarrow 1, \text { as } r \rightarrow \infty \text {. }
$$

For if

then

$$
\lim _{r \rightarrow \infty} \frac{\log \log M(r)}{\log r}<1,
$$

$$
\lim _{r \rightarrow \infty} \frac{(\log r)^{2} \log M(r)}{r}=0,
$$

and so, by Theorem $2, f(z)$ has no asymptotic periods.

If $m B>0$, then $m B_{\sigma}>0$ for some $\sigma<1$. It will be shown that this hypothesis leads to a contradiction.

LEMMa 6. If $a>1$ and

$$
G(r)=\sum_{n=1}^{\infty} e^{-n^{\alpha}} r^{n}
$$

then

$$
\frac{\log \log G(r)}{\log \log r} \rightarrow \frac{a}{a-1}, \text { as } r \rightarrow \infty \text {. }
$$

This is well known. It is easy to prove it by calculating the maximum term of (7.2) and using (5.8). 
Let $\tau, \delta$ be positive numbers satisfying the conditions

(7.1) implies that

$$
\sigma<\tau<1, \quad \delta<\min \left(\frac{1}{9}, \frac{1-\tau}{2}\right) .
$$

$$
r^{1-\delta}<\log M(r)<r^{1+\delta}, \quad\left(r \geqq r_{\delta}\right) .
$$

Now define an auxiliary function

$$
F(z)=\sum_{p=1}^{\infty} e^{-c(p)} f\left(2^{2^{p}} z\right)
$$

where

$$
c(p)=2^{(1-\delta) 2^{p+1}-1} .
$$

If $r=|z|=2^{2^{q}}$, (7.4) gives

while

$$
\begin{aligned}
& \max _{0 \leqq \theta \leqq 2 \pi}\left|e^{-c(q)} f\left(2^{2 q} z\right)\right|=e^{-c(q)} M\left(2^{2^{q+1}}\right) \\
& \quad>\exp \left\{-c(q)+2^{(1-\delta) 2^{q+1}}\right\}=e^{c(q)}, \quad\left(q \geqq \dot{q}_{\delta}\right),
\end{aligned}
$$

$$
\begin{aligned}
\max _{0 \leqq \theta \leqq 2 \pi}\left|e^{-c(p)} f\left(2^{2^{p}} z\right)\right| & =e^{-c(p)} M\left(2^{2^{p}+2^{q}}\right) \\
& <\exp \left\{-c(p)+2^{(1+\delta)\left(2^{p}+2^{q}\right)}\right\} .
\end{aligned}
$$

Now if $p<q$,

$$
\begin{aligned}
c(q)+c(p)-2^{(1+\delta)\left(2^{p}+2^{q}\right)} & >c(q)-2^{(1+\delta)\left({ }^{2 p}+2^{q}\right)} \\
& >X^{(2-3 \delta)}-X^{(1+\delta) 2^{p-q}+1+\delta} \\
& >X^{2-4 \delta}, \quad\left(q \geqq q_{\delta}\right),
\end{aligned}
$$

where $X=2^{2 q}$, since

$$
2-3 \delta>\frac{1+\delta}{2}+1+\delta
$$

Again, if $p>q$,

$$
c(q)+c(p)-2^{(1+\delta)\left(2^{p}+2^{q}\right)}>Y_{p}^{2-4 \delta}, \quad\left(q \geqq q_{\delta}\right),
$$

when $Y_{p}=2^{2^{p}}$.

Denoting by $M_{F^{\prime}}(r)$ the maximum modulus of $F^{\prime}(z)$, and writing $\epsilon=2-4 \delta$, these inequalities show that

Hence

$$
\begin{aligned}
M_{F}(r) & >e^{c(q)}\left\{1-q e^{-X^{\epsilon}}-\sum_{p=q+1}^{\infty} e^{-Y_{p}^{e}}\right\}, \quad\left(q \geqq q_{\delta}\right) \\
& >\frac{1}{2} e^{c(q)}, \quad\left(q \geqq q_{\delta}^{\prime}\right) .
\end{aligned}
$$

$$
\begin{aligned}
\log M_{F}(r) & >2^{2(1-\delta) 2^{q}-1}-\log 2 \\
& =\frac{1}{2} r^{2(1-\delta)}-\log 2,
\end{aligned}
$$

so that $F(z)$ is of order $\geqq 2-2 \delta>1$. 
Let $\beta$ be a member of $B_{\sigma}$, so that $\Delta_{\beta}(z)$ is of order $\leqq \sigma$, and

$$
\Delta_{\beta}(z) \mid<K e^{r^{\tau}}, \quad(r \geqq 0) \text {. }
$$

Now, writing $c_{p}=e^{-c(p)}$,

so that

$$
F(z+\beta)-F(z)=\sum_{p=1}^{\infty} c_{p} \sum_{\varepsilon=0}^{Y_{p}-1} \Delta_{\beta}\left(Y_{p} z+s \beta\right),
$$

Hence, by (7.3),

$$
\begin{aligned}
M_{0}(r) & =\max _{|z|=r}\left|F^{\prime}(z+\beta)-F(z)\right| \\
& <K \sum_{p=1}^{\infty} c_{p} Y_{p} \exp \left\{Y_{p}^{\tau}(r+|\beta|)^{\tau}\right\} \\
& <K \sum_{p=1}^{\infty} c_{p} Y_{p} \exp \left(2 Y_{p} r^{\tau}\right) \\
& =K \sum_{p=1}^{\infty} Y_{p} \exp \left\{-\frac{1}{2} Y_{p}^{2-2 \delta}+2 Y_{p} r^{\tau}\right\} \\
& <K \sum_{n=1}^{\infty} \exp \left\{-n^{2-2 \delta}+4 n r^{\tau}\right\} .
\end{aligned}
$$

$$
\varlimsup_{r \rightarrow \infty} \frac{\log \log M_{0}(r)}{\log r^{\tau}} \leqq \frac{2-2 \delta}{1-2 \delta}
$$

or

$$
\varlimsup_{r \rightarrow \infty} \frac{\log \log M_{0}(r)}{\log r} \leqq\left(\frac{2-2 \delta}{1-2 \delta}\right) \tau<2-2 \delta .
$$

Thus $\beta$ is an asymptotic period of $F(z) . \quad F(z)$ is therefore a function of order $>1$ whose $B$-set contains a subset of $B_{\sigma}$ of positive measure, and this is impossible.

§8. THEOREM 3. If $B$ denotes the set of asymptotic periods of an integral function $f(z)$, either

(i) $B$ is null,

or

(ii) $B$ consists of a set of points $k \lambda, k= \pm 1, \pm 2, \ldots$,

or

(iii) $B$ lies on a line through the origin, is everywhere dense, and has measure zero.

It has been seen that $B$ lies on a line through the origin and that $m B=0$. To show that the only possibilities are the cases enumerated above a further lemma is needed.

LEMMA 7. Let $E$ be a non-null linear set of points with the property that if $x, y$ are any members of $E,-x, x+y$ are also members. Then either (a) $E$ consists of a set of points $k \lambda$, or $(b) E$ is everywhere dense. 
If $E$ is not everywhere dense, let $(\alpha, \beta)$ be an interval not containing a member of $E$. Let $\alpha^{\prime}$ be the upper bound of members of $E \leqq a$, and $\beta^{\prime}$ the lower bound of members of $E \geqq \beta$. Then there are no members of $E$ inside the interval $\left(\beta^{\prime}, 2 \beta^{\prime}-a^{\prime}\right)$. For suppose that such a member, $x$, exists. As $\beta^{\prime}$ is a lower bound of members of $E$ there is a member $y$ satisfying the inequality

$$
\beta^{\prime} \leqq y<\frac{x+\beta^{\prime}}{2} \text {. }
$$

By the addition property $2 y-x$ is a member of $E$, and

$$
a^{\prime}<2 \beta^{\prime}-x \leqq 2 y-x<\beta^{\prime},
$$

giving a contradiction. Similarly there are no members of $E$ inside any interval $\left\{\beta^{\prime}+k\left(\beta^{\prime}-\alpha^{\prime}\right), \beta^{\prime}+(k+1)\left(\beta^{\prime}-a^{\prime}\right)\right\}$, and so $E$ consists of the points $\beta^{\prime}+k\left(\beta^{\prime}-a^{\prime}\right), k=0, \pm 1, \ldots$ As the origin is a member of $E$ it is evident that this set is of the form $k \lambda, k=0, \pm 1, \pm 2, \ldots$

Theorem 3 follows on observing that $B$ has the requisite property (except that the origin is excluded).

§9. Examples. Theorem 2 shows that if $\rho<1$, case (i) is the only possibility, and functions of all orders with no asymptotic periods can be constructed by making use of the following result.

If $f(z)$ is any integral function and $n$ an integer $>2, f\left(z^{n}\right)$ has no asymptotic periods.

For if $s=1,2, \ldots, n-1$,

$$
f\left\{\left(z+e^{2 \pi i \delta / n} \beta\right)^{n}\right\}-f\left(z^{n}\right)=\Delta_{\beta}\left(e^{-2 \pi i s / n} z\right),
$$

so that if $\beta$ is an asymptotic period, the numbers $e^{2 \pi i s / n} \beta$ must have the same property. This is impossible, since they are not collinear.

$e^{z}$ is an obvious example of a function of order 1 which falls under case (ii). A method of constructing functions of higher order with but a single sequence of asymptotic periods is suggested by noticing that the gaps in the series (1.2) played an essential part.

THEOREM 4. Let

$$
f(z)=\sum_{n=1}^{\infty} a(n) e^{n i z}
$$

be an integral function of order $\rho, \dot{(1}<\rho<\infty)$, so that

$$
\lim _{n \rightarrow \infty} \frac{\log \log |a(n)|^{-1}}{\log n}=\frac{\rho}{\rho-1} .
$$


If there is a sequence $n_{1}, n_{2}, \ldots$, such that

$$
\begin{gathered}
n_{p+1}-n_{p}<K, \quad(p=1,2, \ldots), \\
\frac{\log \log \left|a\left(n_{p}\right)\right|^{-1}}{\log n_{p}} \rightarrow \frac{\rho}{\rho-1},
\end{gathered}
$$

then $f(z)$ has no asymptotic periods except a set $k \lambda, k= \pm 1, \pm 2, \ldots$ $\lambda$ must be of the form $\frac{2 \pi}{l}$, where $\lambda$ is an integer. If $f(z)$ has other asymptotic periods they must be everywhere dense on the real axis. Let $N$ be an integer $>K$ and $\beta$ a period $<N^{-1}$. If $q$ is any integer there are at least $N$ of the points $k \beta$ between $2 \pi\left(q+\frac{1}{4}\right)$ and $2 \pi\left(q+\frac{3}{4}\right)$, and so at least one of the points $n_{p} \beta$. Hence

Thus

$$
\left|1-e^{i n_{p} \beta}\right| \geqq \sqrt{ } 2, \text { for a sequence } p_{1}, p_{2}, \ldots
$$

$$
\begin{aligned}
& \max _{0 \leqq x \leqq 2 \pi}|f(x-i y+\beta)-f(x-i y)| \\
& \geqq|a(n)| \cdot e^{n y} \cdot\left|e^{i n \beta}-1\right|, \quad(n \geqq 1), \\
& \geqq \sqrt{ } 2|a(n)| e^{n y}, \quad n=m_{\mathrm{1}}, m_{2}, \ldots \\
& =\sqrt{ } 2 \exp \left(-n^{a_{n}}+n y\right),
\end{aligned}
$$

where $a_{n}=\frac{\rho_{n}}{\rho_{n}-1}, \rho_{n} \rightarrow \rho$, and $m_{1}, m_{2}, \ldots$ is a subsequence of $n_{1}, n_{2}, \ldots$

Thus if $n=m_{t}, y=2 n^{\alpha} n-1$,

$$
\max _{0 \leqq x \leqq 2 \pi} \mid f(x-i y+\beta)-f(x-i y) \vdots \geqq \sqrt{ } 2 \exp \left\{\left(\frac{y}{2}\right)^{\rho_{n}}\right\},
$$

so that $\beta$ cannot be an asymptotic period.

Functions of infinite order presenting case (ii) can be constructed in the same way, e.g. (9.4) may be replaced by

If

$$
\frac{\log \left|a\left(n_{p}\right)\right|^{-1}}{n_{p} \log n_{p}} \rightarrow \alpha>0
$$

$$
\lim _{n \rightarrow \infty} \frac{\log \log \left|c_{n}\right|^{-1}}{n \log n}=\frac{\rho}{\rho-1}, \quad(1<\rho<\infty)
$$

the series

$$
\sum_{n=1}^{\infty} c_{n} e^{2 \pi n ! i z}
$$

defines a function of order $\rho$ having every rational number as an asymptotic period. The construction breaks down if $\rho=1$ and I 
have been unable to find an example of a function of order 1 which falls under case (iii). It may well be that no such function exists.

To sum up:

Case (i). Functions of all orders exist.

Case (ii). Functions of all orders $\rho$ in $(1 \leqq \rho \leqq \infty)$ exist.

Case (iii). Functions of all orders $\rho$ in $(1<\rho \leqq \infty)$ exist.

\section{REFERENCES.}

1. C. Guichard, "Sur la résolution de l'équation aux différences finies $G(x+1)-G(x)=H(x), "$ Annales Sci. de l'Ecole Normale, 4 (1887), 361-380.

2. N. E. Nörlund, Sur la "somme" d'une fonction (Paris, 1927).

3. G. Pólya u. G. Szegö, Aufgaben und Lehrsätze aus der Analysis, I, (Berlin, 1925).

4. G. Valiron, Integral Functions (Toulouse, 1923). 